\title{
Erratum to: Enzyme replacement therapy for mucopolysaccharidosis VI-experience in Taiwan
}

\author{
Hsiang-Yu Lin • Ming-Ren Chen • Chih-Kuang Chuang • Chih-Ping Chen • \\ Dar-Shong Lin • Yin-Hsiu Chien • Yu-Yuan Ke • Fuu-Jen Tsai • Hui-Ping Pan • \\ Shio-Jean Lin • Wuh-Liang Hwu • Dau-Ming Niu • Ni-Chung Lee • Shuan-Pei Lin
}

Published online: 3 November 2010

(C) SSIEM and Springer 2010

Erratum to: J Inherit Metab Dis

DOI:10.1007/s20545-101-9212-5

During the production process, unfortunately, the following information was erroneously omitted from the first page of the article:

Authors Hsiang-Yu Lin, Ming-Ren Chen, and Chih-Kuang Chuang contributed equally to this work.

The online version of the original article can be found at http://dx.doi. org/10.1007/s10545-010-9212-5.

H.-Y. Lin · M.-R. Chen · D.-S. Lin · S.-P. Lin $(\bowtie)$

Department of Pediatrics, Mackay Memorial Hospital,

No.92, Sec. 2 Chung-Shan North Road,

Taipei 10449, Taiwan

e-mail: zsplin@ms2.mmh.org.tw

H.-Y. Lin · C.-K. Chuang • C.-P. Chen · D.-S. Lin · S.-P. Lin

Department of Medical Research, Mackay Memorial Hospital,

Taipei, Taiwan

H.-Y. Lin · M.-R. Chen • D.-S. Lin • S.-P. Lin

Mackay Medicine, Nursing and Management College,

Taipei, Taiwan

H.-Y. Lin • D.-M. Niu

Institute of Clinical Medicine, National Yang-Ming University,

Taipei, Taiwan

C.-K. Chuang

Medical College, Fu-Jen Catholic University,

Taipei, Taiwan

C.-K. Chuang

Institute of Biotechnology,

National Taipei University of Technology,

Taipei, Taiwan

C.-P. Chen

Department of Obstetrics and Gynecology,

Mackay Memorial Hospital,

Taipei, Taiwan

Y.-H. Chien • W.-L. Hwu • N.-C. Lee

Department of Pediatrics, National Taiwan University Hospital, Taipei, Taiwan

Y.-Y. Ke

Department of Pediatrics, Changhua Christian Hospital,

Changhua, Taiwan

\section{F.-J. Tsai}

Department of Pediatrics, China Medical University Hospital, Taichung, Taiwan

H.-P. Pan

Genetic Center, National Cheng Kung University Hospital,

Tainan, Taiwan

\section{S.-J. Lin}

Department of Pediatrics, National Cheng Kung University

Hospital,

Tainan, Taiwan

D.-M. Niu

Department of Pediatrics, Taipei Veterans General Hospital, Taipei, Taiwan

S.-P. Lin

Department of Infant and Child Care,

National Taipei College of Nursing,

Taipei, Taiwan 